\title{
Alfred Bergmann zum 13. Juli 2018
}

Holger Altmeppen

Gregor Bachmann

WALter Bayer

Falk Bernau

Manfred Born

Moritz BrinkMANN

Andreas Cahn

Matthias Casper

Meinrad Dreher

INGo Drescher

Holger Fleischer

Barbara Grüneberg

Barbara Grunewald

Mathias Habersack

STEPhan HaRbarth

Heribert Heckschen

Andreas Heidinger

JOACHIM HENNRICHS

Martin Henssler

Godehard Kayser

Roger Kiem

Detlef Kleindiek

Michael Kling

JENS KOCH

JOACHim Kummer

Katja Langenbucher

Dieter Leuering
JAN LIEDER

Hanno Merkt

Peter O. Mülbert

Andreas Pentz

Markus Roth

INGO SAENGER

Volker Sander

Carsten SchäFER

Jessica Schmidt

KARSTEN SCHMIdT

Sven H. Schneider

Uwe H. SCHNEIDER

Ulrich SeIbert

Christoph H. Seibt

Gerald Spindler

LuTZ STROHN

Christoph Teichmann

Rüdiger VeIL

Dirk A. Verse

EBERHARd VetTer

JOCHEN VETTER

Frauke WedemanN

Simon Weiler

Johannes Wertenbruch

Heinz Wöstmann

Hildegard Ziemons 
